PART 7

U GEMINORUM STARS 


\title{
U GEMINORUM-TYPE STARS
}

\author{
V. G. GORBATSKY \\ Leningrad University Observatory
}

\begin{abstract}
The light variations, properties of U Gem binary systems, outbursts of U Gem systems, and evolution and related problems are discussed.

Recent high-speed photometry of $\mathrm{Z}$ Cha is mentioned in an addendum.
\end{abstract}

\section{The Light Variations}

The main distinguishing feature of $U$ Gem-type stars is their cyclical variability. The brightness of a star during the outburst increases rapidly by $2^{m}$ to $4^{m}$. After some period of constancy the brightness falls to the original value. The light curves of outbursts for the same star may be very different from one to to the other, as well as the time interval between outbursts. The diversity of $U$ Gem-type stars by the lengths of a cycle, amplitudes of outbursts and the shape of the light curve, makes it very difficult to classify a star as belonging to that type. Long series of observations are needed to obtain definite results. The Catalogue of Variable Stars (Kukarkin, et al., 1969/71) contains about 200 stars which belong to this type.

Different determinations of the absolute magnitudes of $U$ Gem-type stars show a large discrepancy. The trigonometric parallax of the brightest star, SS Cyg, leads to $M_{v}=+9^{m} .5$ at minimum light (Strand, 1948), but statistical parallaxes give a mean value $M_{\mathrm{pg}}=+7^{m} \cdot 5 \pm 0.7$ (Kraft and Luyten, 1965). Of course, a considerable dispersion in absolute magnitude may exist.

The total number of $U$ Gem-type stars in the galaxy is estimated to be in the limits

$$
10^{7} \leqq N \leqq 10^{8}
$$

depending on the assumed value of the luminosity. In any case U Gem-type stars are one of the most numerous in the galaxy. In addition, the study of U Gem stars is important due to their connection with novae on one side and with W UMa stars on the other side. The name 'dwarf novae' used often for $U$ Gem stars is misleading. The observed properties of outbursts of $U$ Gem stars are different from nova outbursts, and there is nothing in common with the mechanisms of that process.

Although detailed photometric and spectroscopic data are available only for several of the brightest stars of the type considered - SS Cyg, U Gem, SS Aur - the fragmentary data on the other stars make the picture somewhat clearer. The discussion of the most important observations as well as the numerous examples of light curves can be found in a monograph by Glasby (1970).

The periods of $U$ Gem-type stars are in the interval

$$
10^{\mathrm{d}} \leqq P_{\mathrm{c}} \leqq 300^{\mathrm{d}}
$$


and the photographic amplitudes of outbursts are between

$$
1^{m} \leqq A_{\mathrm{pg}} \leqq 5^{m}
$$

The values for $P_{\mathrm{c}}$ for the same star may differ to $10 \%$, but averaged over a very long time the value $P_{\mathrm{c}}$ is characteristic to the star given. Amplitudes of individual outbursts also differ from one to the other, and there is a well known correlation between the amplitude and the length of the preceding cycle found by Parenago and Kukarkin (1934). This correlation has the form

$$
A_{v}=b_{\mathrm{c}}+1^{m} .85 \lg P_{\mathrm{c}} \text {. }
$$

We shall come back later to the physical background of this correlation.

The duration of outbursts and the rate of the rise to the maximum change from cycle to cycle. The time of constant brightness at maximum may be short, $1^{d}$ to $2^{d}$, or long, $10^{\mathrm{d}}$ to $15^{\mathrm{d}}$, and more. The decline of the brightness takes more time than the increase.

The space distribution of $U$ Gem stars is characteristic to an intermediate-star subsystem. The mean value of the $z$ coordinate is equal to $\langle z\rangle=60 \mathrm{pc}$ (Kraft, 1965), but one must mention some indications of the presence of $U$ Gem stars at high galactic latitudes and in the halo (Haro, 1965). The space velocities are also characteristic for intermediate population stars.

The data on the small subgroup of $Z$ Cam-type stars are of great importance to the understanding of the nature of $U$ Gem stars. Sometimes the brightness of $Z$ Cam drops to an intermediate level and keeps this level for a long time (in some cases during several months and up to a year). Then the brightness decreases to the normal minimum level and the star remains in this state till the next outburst. Owing to the low brightness there are only scanty data on the spectrum of $\mathrm{Z} \mathrm{Cam}$, the most luminous star of this subgroup. Its magnitude at minimum is $14^{m} .5$.

The U Gem stars are variable also between times of outburst. The latest studies have shown that very fast brightness fluctuations are occurring incessantly in all observed stars of this type (Warner and Robinson, 1972). The amplitudes of the fluctuations are of the order of 0.01 to 0.1 and the time scale is determined only by the time resolution power of the equipment used. Because of it the real minimum of brightness cannot be established and one may speak only about a mean minimum level. The brightness fluctuations at minimum are of the same character as in the case of old novae.

In some cases quasi-periodical oscillations of small amplitude are superposed on a very broad power spectrum of brightness fluctuations. The periods of oscillations are of the order of one minute and the amplitudes of about 0.01 . Up to 1972 such oscillations were only found in the old nova DQ Her, but recently similar observations (Warner and Robinson, 1972) were made in several U Gem stars, including Z Cam. The oscillation periods are not strictly constant, as well as the amplitudes. Sometimes the time scale of the variations is very short, $10 \mathrm{~min}$ to $20 \mathrm{~min}$.

The determination of the energy distribution in the continuous spectrum was 
possible up to now only for SS Cyg. At the brightness minima it corresponds to a colour temperature (in the region of $\mathrm{H} \alpha$ and $\mathrm{H} \beta$ ) of the order of $5000^{\circ}$. At the same time one can observe the Balmer jump in emission. At maximum the colour temperature is higher. It reaches $10000^{\circ}$ to $12000^{\circ}$ and the Balmer jump is not in emission. Multicolour observations of SS Cyg and of some other U Gem stars yielded about $U-B \approx-1.0, B-V \approx 0.1$ to 0.3 at minimum.

Some of the known U Gem stars including $U$ Gem itself are eclipsing variables whose orbital periods are several hours. For many reasons one can assert that all U Gem stars are close binary systems. They consist of a dwarf star belonging to a late spectral class ( $G$ to $K$ ) and presumably a white dwarf component. The light curves of eclipsing U Gem stars have many peculiarities, in particular, there is a 'hump' or 'shoulder' preceding the primary light minimum. Usually the secondary minimum is indistinct and even invisible. The depth of the primary minimum is very shallow at the time of the outburst.

One of the most important problems in variable-star physics is the mechanism of the brightness variability. As for the small brightness fluctuations their nature is now clearly understood. Also, one can make some definite conclusions on the mechanism of the outbursts although their complete explanation is a matter of future studies. Beyond all questions, most of the phenomena observed in $U$ Gem stars are not only strongly influenced with the fact of duplicity but this fact seems to be the main cause of variability. Therefore, les us consider the structure of $U$ Gem type close binaries in detail.

\section{The Properties of U Gem-Type Binary Systems}

The duplicity of SS Cyg and other U Gem stars is confirmed from spectroscopic data. During the minimum of a U Gem star the line spectrum consists mainly of broad and intense hydrogen emission lines. These lines are formed in the gaseous disc that exists around the white dwarf component. Their broadening is caused by rapid rotation of the disc and the line width approximately corresponds to the double rotation velocity on the disc's edge. The gas motion in a disc is almost circular Keplerian but owing to turbulent viscosity the gas is accreted on the star's surface (Gorbatsky, 1965); without supply of gas to the disc it would vanish in several days time (Gorbatsky, 1969). The quasi-stationary disc must be fed by the gaseous stream flowing from the other component. The stream is mainly formed in the vicinity of the Lagrangian point $L_{1}$ and its velocity is great enough when it collides with the disc. Let us deduce some quantities that describe the systems of the type considered and the gaseous jets. All these values are estimated from observational data.

The distance between the centers of the

$$
\begin{aligned}
a & =(1 \text { to } 2) R_{\odot} \\
R_{\mathrm{d}} & =(0.2 \text { to } 0.3) \cdot a \\
\Delta r_{\mathrm{d}} & =(0.1 \text { to } 0.3) R_{\mathrm{d}} \\
\bar{\varrho}_{\mathrm{d}} & =\left(10^{-10} \text { to } 10^{-11}\right) \mathrm{g} \mathrm{cm}^{-3}
\end{aligned}
$$$$
\text { components }
$$$$
\text { the radius of a disc }
$$$$
\text { the thickness of a disc }
$$$$
\text { the mean density in a disc }
$$ 
the velocity of the gaseous jet, when entering $v \approx 5 \times 10^{7} \mathrm{~cm} \mathrm{~s}^{-1}$ the disc

the density in the jet at the same time $\quad \varrho_{j}=\left(10^{-12}\right.$ to $\left.10^{-13}\right) \mathrm{g} \mathrm{cm}^{-3}$

Firstly, it can be concluded on the basis of these estimates that the mass of the disc is very small as compared to the total mass of the binary system $\left(<10^{-10} \mathfrak{M}_{\odot}\right)$. Secondly, the energy of the jet motion is not small as compared to the total luminosity of the system.

Using the data given, one can estimate the thermal energy released during the impact of the gaseous jet with the disc. It is approximately equal to $10^{31} \mathrm{erg} \mathrm{s}^{-1}$; that is of the same order as the luminosity. This energy is radiated mainly from the small region of the disc - the so-called 'hot spot'. The radiation emitted by the gaseous stream and in particular by the hot spot, significantly influences the light curve. The presence of a hump before the primary minimum in the case of eclipsing $U$ Gem systems is due to emission from the hot spot. When determining the parameters of systems from photometrical data this must be taken into account.

The jet flowing to the disc must be turbulent and therefore inhomogeneous. Because of density and velocity fluctuations in the jet the energy output from the hot spot region changes in scale, as determined by the size of the fluctuations. It causes the small and rapid oscillations in the observed radiation (Gorbatsky, 1969). The numerous observations of brightness fluctuations of $U$ Gem using high resolution in time (Warner and Nather, 1971) have confirmed this model. One may assert that the presence of small rapid fluctuations of brightness is the sufficient condition to attribute this object to the class of close binary systems having gaseous jets.

As for periodical brightness fluctuations of small amplitude they can also be explained by the impact of a gaseous jet with a disc. In the impact region a standing oscillating shock front must exist (Taranov, 1972). The calculated period and amplitude of shock oscillations are in good agreement with the observed ones.

Other hypotheses were proposed to explain the small periodic oscillations of brightness of some $U$ Gem stars. According to one of them they are the result of nonradial oscillations (in so-called g-mode) of the white dwarf component (Warner and Robinson, 1973). But as the period of the observed fluctuation is variable, this explanation seems highly doubtful. The explanation of periodical fluctuations by the presence of a 'hot spot' on the internal surface of a disc (Bath, 1973) is in contradiction to many observational data. In particular, due to the opaqueness of a disc in optical frequencies we cannot see its regions near the surface of a star.

A hot spot is a region of high ionization and of intense UV radiation. Due to rotation the ionized gas is distributed along the side surface of a disc. Therefore, the temperature in the outer layers of a disc must be high enough, as for example, in the case of RW Tri (Ivanov, 1969). The emission lines are formed not only in the hot spot but in the whole outer region of the disc. The emission lines which originate in the luminous ring have specific profiles consisting of two components. Such profiles are observed in systems having great enough values of $i\left(70^{\circ}\right.$ to $\left.90^{\circ}\right)$. In other cases emission lines are single but they are widened by rotation of the disc. The discs are opaque in 
Balmer-line frequencies and a more elaborate theory of line formation must be used. The results of calculations of the line as well as continuous spectrum intensities are in good agreement with the data of observations of $U$ Gem stars. It is possible to estimate the density of the disc from spectroscopical data. Such estimates have given values of the order of $10^{-11} \mathrm{~g} \mathrm{~cm}^{-3}$ in accordance with determinations on dynamical grounds (Gorbatsky, 1952).

From data on line widths one can find also the mass of the white dwarf component. The mass of the main stars in U Gem-type systems proved to be near the mass limit for white dwarfs, viz., $1.2 \mathfrak{M}_{\odot}$ (Warner, 1973).

Let us say some words on the properties of the secondary stars in U Gem systems. According to the mass ratio $(q)$ determinations for SS Cyg and several other systems, we can adopt the value $q=1$ and consequently $\mathfrak{M}_{2} \approx \mathfrak{M}_{\odot}$ with an uncertainty of about $50 \%$. The spectrum of the secondary (which is visible in SS Cyg and other systems showing no eclipses), is of type $\mathrm{dG}$ or $\mathrm{dK}$. In eclipsing $\mathrm{U}$ Gem systems the spectrum of the secondary as a rule cannot be detected.

The main fraction of the observed optical radiation belongs to the disc-like envelope, and the secondary is emitting only a small part of the total light. Its absolute magnitude $M_{v} \geqslant+9^{m}$ at brightness minima and evidently the secondary is very underluminous during this time interval.

There is no direct evidence for the supposition of a filling up of the Roche lobe by the secondary star. Invisibility of the secondary spectrum in eclipsing systems makes the exact determination of the mass ratio and also of the dimensions of the Roche lobe impossible. The conclusion of such a filling up is inferred usually from the observed fact of mass loss and jet formation near the point $L_{1}$.

However, the filling up of the Roche limit is not the necessary condition of outflow of matter from a star. The gas may flow from the star which is not filling up its Roche limit when the angular velocity of orbital motion $\omega_{0}$ does not coincide with the velocity of axial rotation $\omega_{a}$. The recent calculations of effects of dynamical tides (Koroviakovsky, 1972) has shown that if the star's radius is by $30 \%$ smaller than the dimensions of the Roche lobe and $\omega_{a}=2 \omega_{0}$, the outflow is strong enough to explain the gaseous jets as observed in U Gem systems.

\section{Outbursts of $\mathbf{U}$ Gem Stars}

Faintness of $U$ Gem systems even at light maximum makes the spectral observations of outbursts very difficult, and, hence, hypotheses on the nature of outbursts of $U$ Gem stars were based up to 1950 mainly on visual photometry. Superficial resemblance of $U$ Gem-type light curves to light curves of novae led some investigators to the notion on the similarity of outbursts in both cases. It was assumed that only the scale of phenomena is different.

However, from the scanty data of spectrophotometry of SS Cyg the preliminary conclusion was inferred that the outbursts are mainly caused by increase of the surface temperature of a star (Vorontsov-Velyaminov, 1948). Quantitative analysis (Gorbat- 
sky, 1952) of the continuous spectrum of SS Cyg during outburst (Hinderer, 1949) confirmed this conclusion. It was shown that the star had a surface temperature at light maximum about $10000^{\circ}$ to $12000^{\circ} \mathrm{K}$, and at light minima a much lower temperature, $4000^{\circ}$ to $5000^{\circ} \mathrm{K}$. The radius does not change considerably during the outbursts. As found later SS Cyg is a binary system and this implies that the site of outburst is the cold dwarf companion star. Observed changes in the line spectrum and, in particular, the appearance of broad and strong hydrogen absorption lines, characteristic for A stars (Zuckermann, 1961), are concordant with the notion of the heating of the stellar surface as the cause of the outburst.

The observations of outbursts of the eclipsing system U Gem by Krzeminsky (1965) have given very valuable and up to date uncontended information. It was found that during the brightness increase the primary minimum became very shallow and the eclipse lasted longer. It was inferred from this that the observed increase of brightness was caused mainly by the brightening and simultaneous small expansion of the secondary star. Thus, the picture of the outburst derived from photometric data turns out to be in full agreement with the model obtained from spectroscopic studies.

It was shown later (Smak, 1971) that the locating of the outburst of U Gem in the cold companion is not a necessary corollary from Krzeminski's observations. The primary minimum is caused by the eclipse of a hot spot on the disc's edge. Should the orbital inclination be small enough $\left(i \leqq 70^{\circ}\right)$, then, the white dwarf remains outside the eclipse. Then, one can account the outburst to the increasing luminosity of the primary star not coming in contradiction with Krzeminski's results. Although existing photometric data on $U$ Gem permit both alternative conclusions on the location of the outburst, spectroscopical data are in favour of the cold star as the main source of outbursts. One may mention again that the picture of a nova outburst occurring, as is it usually suggested, in the white dwarf component is very different from the observed U Gem type outbursts.

One can verify Krzeminsky's conclusion by having the light curve of U Gem during the whole period of enhanced luminosity. Let the inclination be great enough to eclipse the hot spot. The disc is opaque to optical radiation and therefore it eclipses partially the secondary. In the case of a considerable increase of brightness of this component there must be a dip on the light curve near phase 0.5 . One may suspect such a dip from the data available (Krzeminsky, 1965) but more complete observations are badly needed.

The observed peculiaritics of $Z$ Cam stars are most important to elucidate the question of the causes of $U$ Gem outbursts. The brightness of such a star after the outburst falls to an intermediate value and may stay on this level very long - during a year and even more. Obviously it can not be considered as a prolonged outburst. Most likely, this level is the normal state in relation to the energy production. The 'plateau' (or 'step') on the light curve ends always with a decline of the brightness to minimum. It follows from these facts that the energy of the outburst is the stored energy produced by internal sources.

There is no sharp limit between U Gem and Z Cam stars. The latter often show 
outbursts of $U$ Gem type and the classical $U$ Gem star may have incidentally the variability of the $\mathrm{Z}$ Cam type as it was demonstrated in the case of SS Aur (Hurless, 1973). Therefore one may assume that the mechanisms of outbursts are identical in both cases. The brightness increase is due to the immediate outcome of stored energy on the stellar surface. The observed cycle-amplitude relation may be simply obtained by this assumption (Gorbatsky, 1971).

As the energy released during U Gem type outbursts is the stored energy from internal sources there is no necessity to sưggest a surplus energy sources. According to existing theory the nova outburst is caused by thermal runaway in the hydrogen external layers of white dwarfs. Evidently this phenomenon is completely different from a U Gem outburst.

On the grounds stated above we consider the location of the outburst in the cold dwarf conpanion as the most probable one. There were several papers concerning the thermal instability of the cold dwarf secondary in close binary systems. As the observed intermediate luminosity of the companion corresponds to a star of $\mathrm{F}$ or $\mathrm{G}$ type an outer convective zone must exist. The instability of the convective zone may play an important role in the origin of ourbursts. However, a simple model of a star expanding beyond its Roche lobe and losing mass until the hot regions of a star become visible (Paczińsky, 1965) is not consistent with observational data. In more elaborate models by Osaki (1970) and by Bath (1972) the outbursts also are connected with the instability of the convective zone but an intense mass loss during outburst following from these theories is in contradiction with the observations.

It seems that the notion of instability of the convective zone as the cause of the $\mathrm{U}$ Gem-type outburst is correct. The main factor leading to storing of energy may be the secular instability of the convective flux (Gorbatsky, 1971). According to the theory of non-stable convection in a nonsynchronously rotating component of a close binary system (Ivanov, 1972) the flux of energy transported by convection may be lowered due to variations in gravity. Therefore, the energy produced by internal sources may be stored near the inner boundary of the convective zone. The time of growth of the instability is of the order of $10^{7} \mathrm{~s}$ and corresponds to the observed duration of the cycle of $U$ Gem stars.

A detailed picture of an outburst is not developed in any theory. Such development will not be an easy work because of very complex phenomena occurring in a medium in which optical and dynamical properties are depending on time.

\section{The Evolution of $\mathbf{U}$ Gem Stars and Related Problems}

The conspicuous feature of many $U$ Gem stars is the change of the orbital period. As it was recently found, the changes are not monotonous (Mumford, 1970) and, therefore, they cannot be explained as the result of mass loss or of exchange of matter between components. It was proposed lately (Smak, 1972) that the mass of a disc-like envelope is great enough to provide a considerable part of the complete momentum of a binary system. The period variability was attributed to changes of the momentum 
of the disc. However, to explain the observations the mass of a disc must be larger than $10^{-5} \mathfrak{M}_{\odot}$. Such a high value of mass is unacceptable on observational grounds as well as on theoretical considerations (Gorbatsky, 1974). Let us mention the value of the disc's mass cited above (Section 2) $\left(10^{-11}\right.$ to $\left.10^{-10}\right) \mathfrak{M}_{\odot}$. The influence of momentum of such an envelope on the orbital period is negligibly small. It seems that the most probable cause of observed period change of $U$ Gem stars is the presence of a third body in a system. One must take into account also the possibility of errors in the $\mathrm{O}-\mathrm{C}$ curve on account of insufficiently precise determinations of the instant of light minima (Breinforst et al., 1973). It may give a spurious effect on the period variability.

The mass loss from U Gem systems does not exceed $10^{-9} \mathfrak{M}_{\odot} \mathrm{yr}^{-1}$ and it cannot lead to a rapid evolution of the internal structure of the components. However, the total output of mass throughout the galaxy may be of a value of $(0.01$ to 0.1$) \mathfrak{M}_{\odot} \mathrm{yr}^{-1}$. Therefore U Gem stars may be one of the important contributors of mass to the interstellar gas. But their role in the evolution of the interstellar medium is not limited to this effect. The output of energy from hot spots on disc-like envelopes may reach to $10^{40}$ to $10^{41} \mathrm{erg} \mathrm{s}^{-1}$. This energy being emitted mainly in the UV region is not very small as compared to the total UV radiation from hot stars in the galaxy. In the calculations of the energy balance for interstellar media the radiation of the hot spots must be taken into account.

One of the most interesting questions, the origin of $U$ Gem systems, is closely connected with the general problem of the origin of close binary systems. There was some progress in the study of this problem during the last decade and now we are able to understand better the history of $U$ Gem systems.

Some years ago the hypothesis had been suggested by Kraft and Sahade for U Gem systems as the descendants from W UMa systems. Their conclusion was based mainly on the similarity between the space distributions and also of the proper motions of the U Gem and W UMa systems. The mass loss was indicated as the main cause of the transformation of a W UMa system to a U Gem system. The consequent study of space distributions and of proper motions of both types of systems on a greater scale has corroborated their similarity in these relations. (Kraft, 1965) But as it is well known W UMa systems are very different from U Gem systems. W UMa systems are nearly contact systems and their components are F or G stars located on the HR diagram near the main sequence while one component of a $U$ Gem system is an evolved and very dense star, presumably a white dwarf. It is assumed usually that W UMa systems are on the zero-age main sequence (Roxburgh, 1966). However, the star of small mass $\left(=\mathfrak{M}_{\odot}\right)$ and of low luminosity cannot evolve to the white dwarf stage in a short time. It is important to mention that white dwarf components of $U$ Gem systems have masses of 1 to $1.2 \mathfrak{M}_{\odot}$ and consequently would be evolved from $\mathrm{F}$ or $\mathrm{G}$ stars without considerable mass loss. These circumstances induce the revision of the current view on components of W UMa stars as being zero-age main sequence stars.

One may suggest that W UMa stars have originated from a rapidly rotating core of an evolved giant star in the process of fission (Gorbatsky, 1975). As some model calculations have shown, it is possible that on certain stages of evolution, momentum is 
not transferred from core to envelope (Kippenhahn, 1970). In such a case the contracting core must increase its angular velocity and may reach the limit of rotational instability. Exact calculations on the fission cannot be made at the present time but the estimates made yielded properties of the configuration that might form a W UMa system. Such a configuration is very similar to the core of a 5 to $7 \mathfrak{M}_{\odot}$ mass star in the stage that immediately precedes helium burning.

The time scale of the fission is unknown but there are some reasons to assume that it is of the same order as the thermal scale, $10^{6}$ to $10^{7} \mathrm{yr}$. Then, the fission should not violate the stellar equilibrium so strongly that it can be observed. However, mass loss from a star may begin most probably in a pulsational form. Taking the mass loss rate $10^{-7}$ to $10^{-8} \mathrm{M}_{\odot} \mathrm{yr}^{-1}$ we must have after lapse of $10^{8} \mathrm{yr}$ a W UMa system. Both components of such a system consist mainly of helium and are enclosed in a hydrogen envelope of comparatively small mass that was captured during the fission. If the more massive component evolves in the Kelvin scale and loses simultaneously its hydrogen envelope the system will be transformed to a U Gem type. There are observational facts favouring this hypothesis. One can mention for instance the overabundance of helium in the W UMa type system TX Cnc noted recently (Whelan et al., 1973).

If the evolutionary way sketched above proves true, the $\mathrm{U}$ Gem systems will turn out as the product of the evolution of massive stars to white dwarfs. It appears that instead of collapse of a massive core there may be sometimes fission of the core to parts having masses below the Chandrasekhar limit.

\section{Addendum}

After this review had been finished an important paper by Warner (1974) came to the knowledge of the author. The paper contains the results of a precise photometry of the U Gem-type eclipsing variable $Z$ Cha during the final stage of the outburst (six days after beginning). At this stage the hump on the light curve before primary minimum was observed and its absolute intensity was several times more than in the normal state. As the hump is caused by radiation from a hot spot its intensity is determined only by the gaseous jet colliding with the disc. Therefore one can conclude that the outflow of matter from the secondary was considerably enhanced at this stage of the outburst. The eclipse at this time was two times wider than normal. If one does not make improbable assumptions it can be explained mainly by an increasing of the dimensions of an eclipsing body. Consequently the secondary is expanding during the outburst. Mass loss is enhanced presumably due to expansion of the star and the luminosity of the disc must be higher. It signifies that the outburst is caused by the instability of the secondary, leading, in particular to more intensive outflow of matter. Such a possibility was suggested earlier (Smak, 1971).

As for the changes of brightness during the outburst, the spectral data (Zuckermann, 1961) as well as the data on colours (Chuvayev, 1962) have shown that the radiation from the disc must be relatively weakened during the first one or two days, and strongly increased radiation of the secondary prevails over the primary. The radiation from 
internal regions of the disc as well as from the hot spot strongly depends on the output of the gas in the jet. If the jet is powerful enough, the radiation from the entire disc may play the main role in the total observed light. It seems that such a situation is realized in the case of $Z$ Cha. The observations considered confirm the fact of the instability of the secondary as the main cause of outburst. They also gave the clue to the understanding of the nature of long outbursts.

\section{References}

Bath, G.: 1972, Astrophys. J. 173, 121.

Bath, G.: 1973, Nature Phys. Sci. 246, 84.

Breinforst, R., Pfleiderer, J., Reinhardt, M., and Karinie, M.: 1973, Astron. Astrophys. 22, 239.

Chuvagev, K. K.: 1962, Izv. Krymsk. Astrofiz. Obs. 28, 141.

Glasby, J.: 1970, The Dwarf Novae, London.

Gorbatsky, V. G.: 1952, Astron. Zh. 29, 682.

Gorbatsky, V. G.: 1965, Trudy Astron. Obs. Leningr. Univ. 22, 16.

Gorbatsky, V. G.: 1969, in L. Detre (ed.), Non Periodic Phenomena in Variable Stars, Budapest, p. 391.

Gorbatsky, V. G.: 1971, Astron. Zh. 48, 671.

Gorbatsky, V. G.: 1974, Novalike and Nova Stars, 'Nauka', Moscow.

Gorbatsky, V. G.: 1975, Astrophys. Space Sci. (in press).

Haro, G.: 1965, Publ. Univ. of Minn. Obs. First Conference on Blue Stars.

Hinderer, F.: 1949, Astron. Nachr. 277, 193.

Hurless, C. J.: 1973, AAVSO J. 2, 35.

Ivanov, L. N.: 1969, Trudy Astron. Obs. Leningr. Univ. 24, 33.

Ivanov, L. N.: 1972, Vestnik Leningr. Univ. No. 13, 126.

Kippenhahn, R.: 1970, Astron. Astrophys. 5, 155.

Korovyakovsky, Yu. P.: 1972, Izv. Special Astrophys. Obs. 4, 115.

Kraft, R.: 1965, Astrophys. J. 142, 1588.

Kraft, R. and Luyten, W.: 1965, Astrophys. J. 142, 1041.

Krzeminsky, W.: 1965, Astrophys. J. 142, 1051.

Kukarkin, B. V., Kholopov, P. N., Efremov, Yu. N., Kukarkina, N. P., Kurochkin, N. E., Medvedeva, G. I., Perova, N. B., Fedorovich, V. P., and Frolov, M. S.: (1969-1971), General Catalogue of Variable Stars, 3nd ed. I-III, Moscow.

Mumford, G.: 1970, Astrophys. J. 162, 363.

Osaki, J.: 1970, Astrophys. J. 162, 621.

Paczińsky, B.: 1965, Acta Astron. 15, 89.

Parenago, P. P. and Kukarkin, B. V.: 1934, Peremen. Zvezdy 4, 245, 249.

Roxburgh, J.: 1966, Astrophys. J. 143, 111.

Smak, J.: 1971, Acta Astron. 21, 15.

Smak, J.: 1972, Acta Astron. 22, 1.

Strand, K., 1948, Astrophys. J. 107, 106.

Taranov, V. I.: 1971, Astrophysica 7, 295.

Vorontsov-Velyaminov, B. A.: 1948, The Gaseous Nebulae and Novae Stars, Moscow.

Warner, B.: 1973, Monthly Notices Roy. Astron. Soc. 162, 189.

Warner, B.: 1974, Monthly Notices Roy. Astron. Soc. 168, 235.

Warner, B. and Nather, R.: 1971, Monthly Notices Roy. Astron. Soc. 152, 219.

Warner, B. and Robinson, E. L.: 1972, Nature Phys. Sci. 239, 2.

Whelan, J., Worden, S., and Mochnacki, S.: 1973, Astrophys. J. 183, 133.

Zuckermann, M.: 1961, Ann. Astrophys. 24, 431.

\section{DISCUSSION}

M. Friedjung: Could not the variation in the colour of the continuous spectrum be interpreted as due to a contribution of the secondary at minimum light which does not exist at maximum?

V. G. Gorbatsky: The work was based on the analysis of spectra taken by Hinderer.

I do not have specific data. 
$V$. A. Ambartsumian: How long do the standstills at intermediate brightness level (as mentioned in your paper) last in the case of $\mathrm{Z}$ Cam? To what accuracy can we state that the star is constant?

$V$. G. Gorbatsky: In one case the standstill lasted for more than a year and in many other cases it lasted for several months.

I. G. Bochkarev: What energy is emitted by the hot spot at the boundary between the jet and the disc?

$V$. G. Gorbatsky: The total energy emitted by the system is of the order of $10^{31} \mathrm{erg} \mathrm{s}^{-1}$. Since the cold component is usually not seen, all this energy must be attributed to the disc. This concerns the optical radiation.

In the X-ray region, the intensity of the radiation and its spectrum depend on the structure of the standing shock wave at the boundary between the jet and the disc. The estimates are uncertain, but probably the energy must be about $10^{33} \mathrm{erg} \mathrm{s}^{-1}$ (probable range $10^{31}$ to $10^{33} \mathrm{erg} \mathrm{s}^{-1}$ ). The slope of the spectrum is 0.5 or more (the intensity increases when the energy of quanta decreases). The energy of quanta near the low-frequency cutoff of the spectrum depends upon the presence of gas in the system and its numerical value is not yet known. Clearly the X-ray radiation from nearby stars, e.g. SS Cyg, may easily be observed from satellites. The radiation must exist all the time outside the explosions. The situation during an explosion is not clear. The X-ray radiation from $\mathrm{U}$ Gem stars may be responsible for the observed isotropic X-ray background $(h v<1 \mathrm{keV})$.

$N$. Z. Voyhanskaya: At present we have two alternative models for the explosion. Do you think we can distinguish between these models by observing the light curves and the spectral changes?

$V$. G. Gorbatsky: The spectral changes at the beginning of the brightness increase are known; for instance, the appearance of the A type spectrum. However, it's difficult to say something after the beginning of the extensive mass exchange. 